\title{
Investigation of the Nonlinear Optical Response of 3- (Dimethylamino)-7-Aminophenothiazin-5-Ium Chloride Dye
}

\author{
Hussain A. Badran \\ Department of Physics, College of Education, University of Basrah, Basrah, Iraq
}

\begin{abstract}
The nonlinear optical properties of 3-(Dimethylamino)-7-aminophenothiazin-5-ium chloride dye in the solvent chloroform was studied by Diffraction ring technique using cw diode laser at $532 \mathrm{~nm}$. The obtained results for the nonlinear refractive index, $n_{2}$, and the thermo-optic coefficients, $d n / d T$, are found to be of the order of $10^{-7} \mathrm{~cm}^{2} / W$ and $10^{-5} \mathrm{k}^{-1}$ respectively. To solidate the present experimental results the diffraction ring patterns are reproduced theoretically based on a well known simple model. The rings number of each pattern variation with power agree well with the experimental findings. These results indicate that the azo dye is a promising candidate for applications in nonlinear optical devices.
\end{abstract}

Keywords: Nonlinear refractive index, Thermo-optic coefficients, Diffraction ring

\section{INTRODUCTION}

Materials with high third- order nonlinear refractive index are always of large interests for their potential applications on many nonlinear optical devices such as optical limiting, beam flattening, optical switching, weak absorption measurement, spatial dark solution transmission [1-4] and so on.

Changes in refractive index by optical field give rise to a variety of nonlinear phenomena in photoresponsive materials. In the spatial domain, the interplay between divergence of the propagating beam and the nonlinear optical response of the medium can elicit a diverse rang of self-action behaviour such as optical self-trapping, solution formation and spontaneous pattern formation due to modulation instability [5]. A related phenomenon is the spatial self modulation of a coherent beam, which generates a nested array of concentric intensity ring in the far field. Such diffraction ring have been observed in media with thermally-dependent refractive index change[6-9] atomic vapours [10-13], nematic liquid crystals [14-16] ,Kerr media [17,18] ,chromophore- substituted silica [19] and photorefractive crystals [20].

In the present work we presents experimental evidences of observing diffraction pattern in 3(Dimethylamino)-7-aminophenothiazin-5-ium chloride dye with the calculation of the refractive index change, $\Delta n$, effective nonlinear refractive index, $\mathrm{n}_{2}$, and variation of refractive index with temperature , $\mathrm{d} n / \mathrm{dT}$.Using theoretical model based on wave theory we have reproduce the diffraction patterns. The obtained results fit reasonably the experimental one.

\section{DIFFRACTION RING TECHNIQUES}

We can estimate the induced refractive index change, $\Delta n$, and the effective nonlinear refractive index , $n_{2}$, for the preceding data as follows. Because the laser beam used in the experiment has a Gaussian distribution, the relative phase shift , $\Delta \varphi$, suffered by the beam while traversing the sample of thickness, $L$ can be written as [8]:

$$
\Delta \varphi=k L \Delta n
$$

where $k=2 \pi / \lambda$ is the wave vector in vacuum and $\lambda$ is the laser beam wavelength.

The relationship between $\Delta \varphi$ and number of rings, $N$, can be written as [21]:

$$
\Delta \varphi=2 \pi N
$$

The relationship between the total refractive index,$n$, and nonlinear part of the refractive index , $n_{2}$, can be written as follows [22]:

$$
\begin{aligned}
& n=n_{0}+\frac{n_{2}}{2} I \\
& n=n_{0}+\Delta n
\end{aligned}
$$

where $n_{0}$ is the background refractive index.

The thermal lens signal is expressed as the relative change in power [23] 


$$
\begin{gathered}
\theta=\frac{I_{\circ}-I}{I} \\
\theta=\frac{\Delta I}{I}=\frac{\alpha L_{\text {eff }} P}{\lambda K}\left(-\frac{d n}{d T}\right)
\end{gathered}
$$

For the thermal nonlinearity and steady state case, the change nonlinear index, $\Delta n$, can be expressed as [8]:

$$
\Delta n=\frac{d n}{d T} \cdot \frac{I \alpha \omega_{\circ}^{2}}{4 K}
$$

Where $I_{\circ}$ and $I$ are the transmitted power before and after is the formation of the thermal lens respectively. $\alpha$ is the linear absorption coefficient, $L_{\text {eff }}$ is the effective thickness of the sample, $P$ is the laser input power, $d n / d T$ and $K$ are the sample temperature coefficient of refractive index and thermal conductivity, respectively.

By the combination of equations (1-6) one can calculate, $\Delta n, n_{2}$ and $d n / d T$. The Diffraction Ring experiments were performed using a $532 \mathrm{~nm}$ solid state laser beam, which was focused by $+50 \mathrm{~mm}$ focal length lens. The laser beam waist $\omega_{0}$ at the focus is measured to be $21.63 \mu \mathrm{m}$, the linear absorption coefficient $\alpha=2.18 \mathrm{~cm}^{-1}$ and the effective thickness of the sample $L_{\text {eff }}$ is measured to be $0.089 \mathrm{~mm}$. The schematic of the experimental set up used is shown in Fig. 1. A $1 \mathrm{~mm}$ wide optical cell containing the solution of 3(Dimethylamino)-7-aminophenothiazin-5-ium chloride dye in the solvent chloroform is translated across the focal region along the axial direction that is the direction of the propagation laser beam.

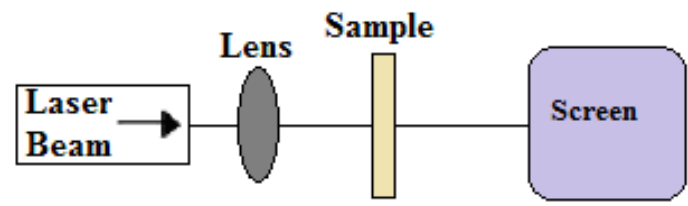

Fig. 1. Schematic diagram of experimental arrangement for the Diffraction Ring measurement.

Figure 2 shows the far-field diffraction ring patterns at different input powers at $0.05 \mathrm{mM}$ concentration. These are photographs of the pattern on a screen placed about $65 \mathrm{~cm}$ behind the sample. The number of rings increases with increasing input power.

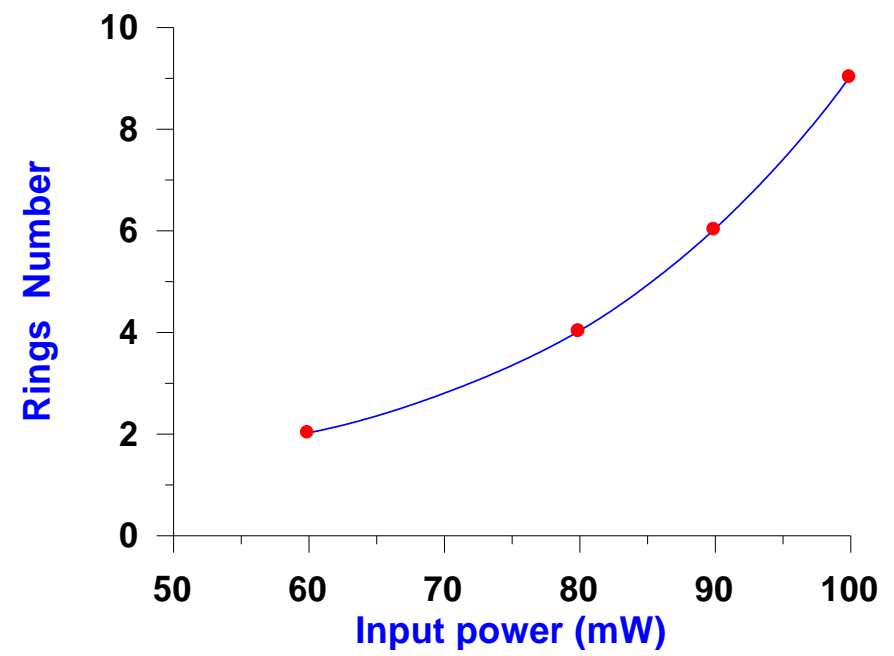

Figure 2: diffraction ring patterns as a function of different input powers for $0.05 \mathrm{mM}$ concentration

Figure 3 shows the number of observed ring as a function of the input power. In our experiment we obtained a maximum ring number of 9 at an input power of $100 \mathrm{~mW}$. Our patterns are quite concentric and sharp. This is because the scattering of a laser beam in solvent, which is caused by the fluctuation of molecular axes. The outermost ring is the strongest of all the rings and is especially wide. We were not able to obtain definite rings at input power levels over that value, probably due to the boil of 3-( Dimethylamino) -7- aminophenothiazin-5-ium 
chloride dye in the solvent chloroform. We also find that the half-cone angle of the contermost ring increases in proportion to the input power, up to $\sim 15 \mathrm{deg}$ in our experiment.

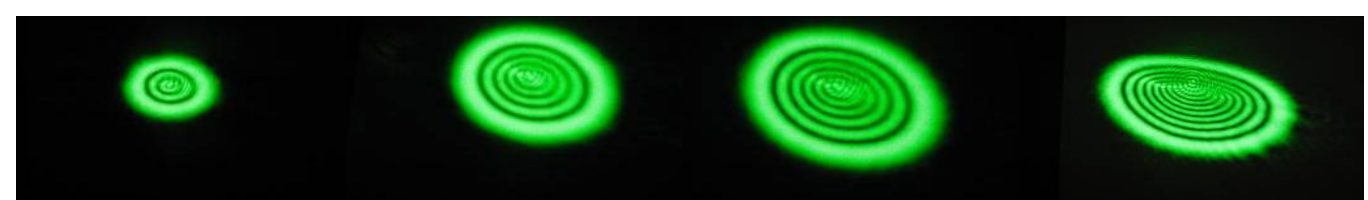

(a) (c) (d)

Fig.3: diffraction ring patterns for the azo dye in the solvent chloroform (a) 2 at 60mW, (b) 4 at $80 \mathrm{~mW}$, (c) 6 at $90 \mathrm{~mW}$, (d) 9 at $100 \mathrm{~mW}$.

As given in Table 1 , the Nonlinear parameters $\left(\beta, \Delta n\right.$, and $\left.n_{2}\right)$ and thermo-optic coefficient,$d n / d T$, increases with increasing the number of rings $N$ and input power for the azo dye solutions at $0.05 \mathrm{mM}$ concentration.

Table 1: Number of rings, phase shift, change in refractive index, nonlinear refractive index and thermo-optic coefficient for the sample.

\begin{tabular}{|c|c|c|c|c|c|}
\hline Power $(\mathrm{mW})$ & $N$ & $\Delta \varphi$ & $\Delta n$ & $n_{2}\left(\mathrm{~cm}^{2} / \mathrm{W}\right)$ & $d n / d T\left(\mathrm{k}^{-1}\right)$ \\
\hline 60 & 2 & 0.219 & $0.206 \times 10^{-4}$ & $0.253 \times 10^{-8}$ & $0.196 \times 10^{-5}$ \\
\hline 80 & 4 & 0.438 & $0.413 \times 10^{-4}$ & $0.379 \times 10^{-8}$ & $0.295 \times 10^{-5}$ \\
\hline 90 & 6 & 0.657 & $0.619 \times 10^{-4}$ & $0.506 \times 10^{-8}$ & $0.393 \times 10^{-5}$ \\
\hline 100 & 9 & 0.985 & $0.929 \times 10^{-4}$ & $0.683 \times 10^{-8}$ & $0.531 \times 10^{-5}$ \\
\hline
\end{tabular}

The high nonlinear optical refractive index compares favourably with that of some representative of third-order nonlinear optical materials, namely, pararosanilin dye in liquid and solid media [24], basic green 1 dye in aqueous solutions [25], oxazine (OX720) and oxazine (OX750) dye in aqueous solutions and in PAA matrix [23], photo polymerizable organo siloxane [22], and organic polymers [26]. These results predict that 3(Dimethylamino)-7-aminophenothiazin-5-ium chloride dye has potential applications for nonlinear optics. For practical use in all-optical switching devices many considerations have been taken into account to investigate the effectiveness of nonlinear materials. This large nonlinear refraction makes of an 3-(Dimethylamino)-7aminophenothiazin-5-ium chloride dye promising for use in all-optical switching devices.

\section{THEORETICAL MODEL OF THE DIFFRACTION RING}

The laser beam used in present work has a Gaussian extent of wavelength $\lambda$. Assuming that the beam propagate along the z-direction and the medium have a length of $L$ with a linear absorption coefficient $\alpha$. At the entrance of the medium which is taken as the origin of the Cartesian coordinates the electric field of the incident laser beam can be written as [27]:

$$
E(r, z)=E\left(0, z_{o}\right) \exp \left[\frac{-r^{2}}{\omega_{\circ}^{2}}\right] \exp \left[\frac{-i k n_{o} r^{2}}{2 R}\right]
$$

where $r$ is the radial coordinate, $z_{0}$ is the coordinate position of the medium, $k$ is the free space wave vector, $\omega_{0}$ is the beam waist at the medium entrance and $R$ the radius of curvature of its wave-front in the corresponding position.

By taking into account the total phase shift, $\varphi$, suffered by the beam during the course of traversing through the medium, the far-field distribution pattern can be obtained considering the free propagation of the optical wave through space, by means of Fraunhofer approximation of the Fresnel- Kirchhoff diffraction integral as [27] :

$$
I(\rho)=\left.I_{o} \int_{0}^{\infty} J_{o}(k \theta r) \exp \left[-\frac{r^{2}}{\omega_{\circ}^{2}}-i \varphi(r)\right] r d r\right|^{2}
$$

Where $J_{\mathrm{o}}(x)$ is the zero-order Bessel function of the first kind, $\theta$ is the far field diffraction angle, $\rho$ is the radial coordinate in the far field observation plane and $I$ 。 can be written as: 


$$
I_{o}=4 \pi^{2}\left|\frac{E\left(0, z_{o}\right) \exp (-\alpha L / 2)}{i \lambda D}\right|^{2}
$$

$D$ is related to the radial coordinate by $\rho=D \theta$.

Using the values of the various parameters given in table 2 and solving equation 6 and 7 we have generated the theoretical results of diffraction pattern shown in fig. 4 for the four chosen input powers.

Table2. Measured and calculated values of the parameters used to generate figure 4.

\begin{tabular}{ll}
\hline Laser beam wavelength $(\lambda)$ & $532 \mathrm{~nm}$ \\
Laser beam waist $\left(\omega_{\circ}\right)$ & $21.63 \mu \mathrm{m}$ \\
Input power $(p)$ & $10-100 \mathrm{mWatt}$ \\
Radius of wave-front $(R)$ & $33 \mathrm{~mm}$ \\
Cell length $(L)$ & $1 \mathrm{~mm}$ \\
Distance from the exit plane $(D)$ & $65 \mathrm{~cm}$ \\
\hline
\end{tabular}

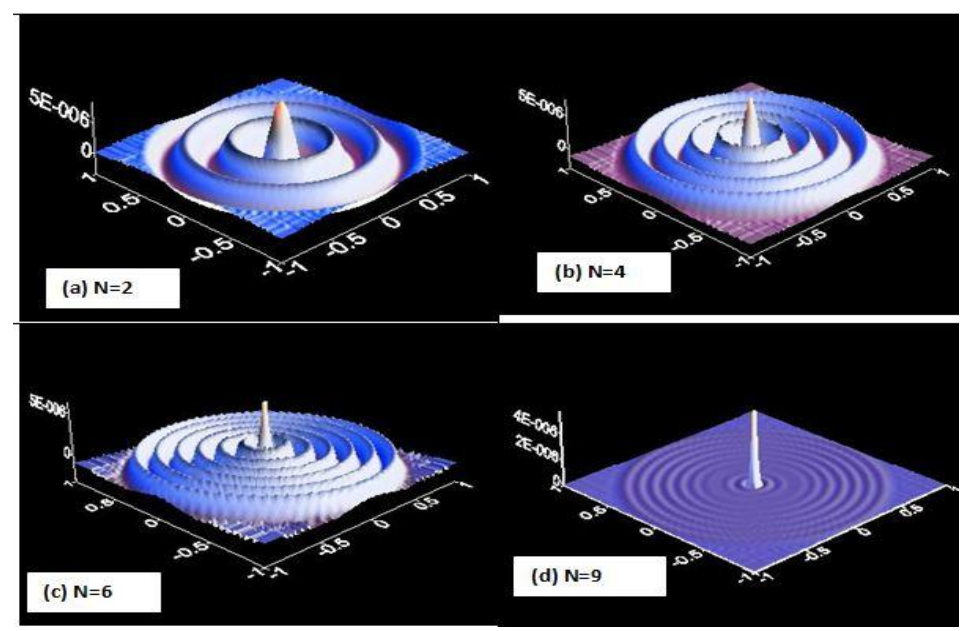

Fig.4: Theoretical results of the diffraction patterns: (a) $\mathrm{P}=60 \mathrm{mWatt}$, (b) $\mathrm{P}=80 \mathrm{mWatt}$, (c) $\mathrm{P}=90 \mathrm{~mW}$ att and (d) $\mathrm{P}=100$ mWatt.

\section{CONCLUSION}

The nonlinear optical properties of 3-(Dimethylamino)-7-aminophenothiazin-5-ium chloride dye in chlorophorm solvent was studied using diffraction pattern technique with a continuous-wave radiation at 532 $\mathrm{nm}$ of an different output power. All the solution samples showed large nonlinear refractive indexice and absorption coefficient of the order of $10^{-8} \mathrm{~cm}^{2} / \mathrm{W}$ and $10^{-3} \mathrm{~cm} / \mathrm{W}$, respectively. Experimental results of ring patterns reinforced theoretically suggest the possibility of using 3-(Dimethylamino)-7-aminophenothiazin-5-ium chloride dye solution in all optical systems .These patterns were generated in 3-(Dimethylamino)-7aminophenothiazin-5-ium chloride dye by the irradiation with visible laser beam of Gaussian extent.The instantaneous formation of rings prove the fast response of this substance. The thermal number of rings observed increases with increasing input power nonlinearly. The stability of the ring patterns suggest the stability of such medium.

\section{REFERENCES}

[1] O. Durand, V. Grolier-Mazza, and R. Frey, Picosecond-resolution study of nonlinear scattering in carbon black [2] suspensions in water and ethanol, Opt. Lett. 23(18), 1998, 1471-1473.

[3] J. Staromlynska, T. J. McKay and P. Wilson, Broadband optical limiting based on excited state absorption in Pt:ethynyl

[4] J. Appl. Phys. 88(4), $2000,1726-1732$.

[5] S.Vijayakumara,A. Adhikarib, B. Kallurayab and K. Chandrasekha rana, $\chi^{3}$ measurements and optical limiting studies of 2chloro-5-nitro-N-[(1E)-phenylmethylidene] benzohydrazide with substituents, Opt. Mater. 31 (11) (2009)1564-1569.

[6] M. Zhengle, Q. Lingling, H. Fei, L. Yang, W. Chen and C. Ya, Thermal-induced nonlinear optical characteristics of ethanol solution doped with silver nanoparticles, Chinese optics letters ,7( 10), 2009, 949-952.

[7] Trill S. and Torruellas W. Spatial solitons (Berlin: Springer, 2001 ).

[8] W. R. Callen, B. G. Huth, and R. H. Pantell. optical patterns of thermally self - defocused light, Appl. Phys. Lett. 11(3), 1967 , 103-105. 
[9] F. W. Dabby, T. K. Gustafson, J. R. Whinnery, Y. Kohanzadeh, and P. L. Kelley, thermally self - induced phase modulation of laser beams, Appl. Phys. Lett. 16 (9), 1970, 362-365 .

[10] K. Ogusu, Y. Kohtani, and H. Shao , Laser-induced diffraction rings from an absorbing solution, Opt. Rev.3(4), 1996, 232234R.G Harrison, L Dambly, Dejin Yu, Weiping Lu, A new self-diffraction pattern formation in defocusing liquid media, Optics Communications, 139 (13), 1997, 69-72 .

[11] D.Grischkowsky, Self-focusing of light by potassium vapor, Phys. Rev. Lett.,24(16) ,1970,886--869 .

[12] C.Wang,Y.Fu,Z.Zhou,Y.Cheng and Z.Zhan Xu, Femtosecond filamentation and supercontinuum generation in silver-nanoparticledoped water, Appl. Phys. Lett. 90 (18), 2007, 181119-181122.

[13] Y. H. Meyer, Multiple conical emission from near resonant laser propagation in dense sodium vapor, Opt. Commun. 34(3), 1980, 439-444.

[14] C. H. Skinner and P. D. Kleiber, Observation of anomalous conical emission from laser-excited barium vapor, Phys. Rev. A 21(1), $1980,151-156$.

[15] S.D. Durbin, S. M. Arakelian and Y. R Shen, Laser-induced diffraction rings from a nematic- liquid-crystal film. Opt Lett. , 6(9) , 1981, 411-413.

[16] N.F. Pilipetski, A.V. Sukhov, N.V. Tabiryan, B.Ya. Zel'dovich. The orientational mechanism of nonlinearity and the self-focusing of He-Ne laser radiation in nematic liquid crystal mesophase (theory and experiment), Opt. Commun,37(4) 1981 , 280-284.

[17] E . Santamato and Y.R Shen, Field curvature effect on the diffraction ring pattern of a laser beam dressed by spatial self- phase modulation in a nematic film .Opt. Lett. 9(12), 1984, 564-566.

[18] P.C.Lee and D.Meisel ,Adsorption and surface-enhanced Raman of dyes on silver and gold sols,J.Phys.Chem. 86 (17), 1982 , 3391-3395.

[19] M. Sheik-Bahae, A.A. Said, T.H. Wei, D.J. Hagan and E.W. Van Stryland, Sensitive measurement of optical nonlinearities using a single beam, IEEE J. Quantum Electron. 26(4), 1990, 760-769.

[20] R. W. Boyd. Nonlinear optics, (2nd ed), (Academic Press, London, 2003) p.220 .

[21] R.C.Kamikawachi, I.Abe, A.S.Pateron, H.J.Kalinowski, M.Muller, J.L. Pinto and J. L. Fabris, , Determination of the thermo-optic coefficient in liquid with fiber bragggrating refractometer Opt. Commun. 281(4), 2008 , 621-625.

[22] A. B. Villafranca and K. Saravanamuttu, Diffraction rings due to spatial self- phase modulation in a photopolymerizable medium, J. Opt. A: Pure Appl. Opt. 11(12),2009, 125202.

[23] K. Milanchian, H. Tajalli, A. Ghanadzadeh Gilani and M.S. Zakerhamidi, Nonlinear optical properties of two oxazine dyes in aqueous solution and polyacrylamide hydrogel using single beam Z-scan, Opt. Mat. 32(1) , 2009, 12-17.

[24] S. Acevedo, M.A. Ranaudo, J.C. Pereira, J. Castillo, A. Fernández, P. Pe'rez and M. Caetano, Thermo-optical studies of asphaltene solutions: evidence for solvent-solute aggregate formation, Fuel 78(9), 1999, 997-1003 .

[25] G. Vinitha, A. Ramalingam, P.K. Palanisamy, Nonlinear studies of Pararosanilin dye in liquid and solid media, Spect. Acta Part A: Mol. and Biom. Spec $68,2007,1-5$.

[26] Q.M. Ali and P.K.Palanisamy, Investigation of nonlinear optical properties of organic dye by z-scan technique using He-Ne laser, Optik $116(11), 2005,515-520$

[27] S.A.Jenekhe,W.Chen,S.Lo and S.R.Flom.Large thired-order optical nonlinearities inorganic polymer superlattices, Appl. Phys. Lett. 57(2), 1990, 126-128.

[28] S. Chávez-Cerda, C. M. Nascimento, M. A. R. C. Alencar, M. G. A. da Silva, M. R. Meneghetti and J. M. Hickmann, Experimental observation of the far field diffraction patterns of divergent and convergent beam in a self-defocusing medium, Annals of Optics, XXIX ENFMC , $2006,1-4$. 